\title{
High-Dose Methamphetamine Acutely Activates the Striatonigral Pathway to Increase Striatal Glutamate and Mediate Long-Term Dopamine Toxicity
}

\author{
Karla A. Mark, ${ }^{1}$ Jean-Jacques Soghomonian, ${ }^{2}$ and Bryan K. Yamamoto ${ }^{1}$ \\ ${ }^{1}$ Laboratory of Neurochemistry, Department of Pharmacology and Experimental Therapeutics, and 2Department of Anatomy and Neurobiology, Boston \\ University School of Medicine, Boston, Massachusetts 02118
}

\begin{abstract}
Methamphetamine (METH) has been shown to increase the extracellular concentrations of both dopamine (DA) and glutamate (GLU) in the striatum. Dopamine, glutamate, or their combined effects have been hypothesized to mediate striatal DA nerve terminal damage. Although it is known that METH releases DA via reverse transport, it is not known how METH increases the release of GLU. We hypothesized that METH increases GLU indirectly via activation of the basal ganglia output pathways. METH increased striatonigral GABAergic transmission, as evidenced by increased striatal GAD65 mRNA expression and extracellular GABA concentrations in substantia nigra pars reticulata $(\mathrm{SNr})$. The METH-induced increase in nigral extracellular GABA concentrations was D1 receptor-dependent because intranigral perfusion of the D1 DA antagonist SCH23390 (10 $\mu \mathrm{M})$ attenuated the METH-induced increase in GABA release in the SNr. Additionally, METH decreased extracellular GABA concentrations in the ventromedial thalamus (VM). Intranigral perfusion of the GABA-A receptor antagonist, bicuculline $(10 \mu \mathrm{M})$, blocked the METH-induced decrease in extracellular GABA in the VM and the METHinduced increase in striatal GLU. Intranigral perfusion of either a DA D1 or GABA-A receptor antagonist during the systemic administrations of METH attenuated the striatal DA depletions when measured 1 week later. These results show that METH enhances D1mediated striatonigral GABAergic transmission (1), which in turn activates GABA-A receptors in the $\mathrm{SNr}(2)$, leading to a decrease in GABAergic nigrothalamic activity (3), an increase in corticostriatal GLU release (4), and a consequent long-term depletion of striatal DA content (5).
\end{abstract}

Key words: GABA; D1; GABA-A; substantia nigra; thalamus; microdialysis

\section{Introduction}

The abuse of the psychostimulant methamphetamine (METH) has grown over the last decade. One major concern of METH abuse is the potential long-term striatal dopaminergic and serotonergic deficits associated with repeated exposure over time. Specifically, studies in rodents and primates show long-term decreases in markers associated with dopamine (DA) and serotonin (5-HT) toxicity, including decreases in monoamine transporter immunoreactivities, tyrosine and tryptophan hydroxylase activities, and terminal degradation (Hotchkiss and Gibb, 1980; Wagner et al., 1980; Ricaurte et al., 1982).

METH acutely increases extracellular concentrations of DA and glutamate (GLU) in the striatum (O'Dell et al., 1991; Stephans and Yamamoto, 1994). The combined effect of DA and GLU release is thought to produce oxidative stress and glutamate-mediated excitoxicity to DA nerve terminals (Sonsalla

\footnotetext{
Received Sept. 1, 2004; revised Nov. 12, 2004; accepted Nov. 13, 2004.

This work was supported by National Institutes of Health Grant DA07606, Department of the Army Grant DAMD 17-99-1-9479, and a gift from Hitachi America.

Correspondence should be addressed to Bryan K. Yamamoto, Department of Pharmacology and Experimental Therapeutics, Boston University School of Medicine, 715 Albany Street, Room L-613, Boston, MA 02118. E-mail: bkyam@bu.edu.

DOI:10.1523/JNEUROSCI.3597-04.2004

Copyright $\odot 2004$ Society for Neuroscience $\quad$ 0270-6474/04/2411449-08\$15.00/0
}

et al., 1989; Nash and Yamamoto, 1992; Yamamoto and Zhu, 1998; LaVoie and Hastings, 1999; Imam et al., 2001). Although METH is known to release DA directly via reverse transport, little is known about the mechanisms of METH-induced GLU release in the striatum.

The glutamatergic innervation of the striatum arises primarily from corticostriatal terminals (Gerfen, 1989; Bellomo et al., 1998). The corticostriatal pathway can be regulated by the output pathways of the basal ganglia involving the nigrothalamic GABAergic and thalamocortical glutamatergic projections (Kaneko and Mizuno, 1988). Specifically, GABAergic projection neurons from the striatum terminate mainly in the substantia nigra pars reticulata $(\mathrm{SNr})$. The $\mathrm{SNr}$ contains a high density of DA D1 receptors, mainly distributed on presynaptic striatonigral terminals (Altar and Hauser, 1987; Martin and Waszczak, 1994; Trevitt et al., 2002) to positively modulate GABA release within the SNr (Reubi et al., 1977, 1978; Kelly et al., 1985; Aceves et al., 1995). This dopaminergic regulation of GABAergic neurotransmission within the $\mathrm{SNr}$ can directly influence the nigrothalamic pathway via postsynaptic GABA-A receptors distributed on the soma of nigrothalamic projections (Nicholson et al., 1995) and consequently affect thalamocortical activity (Timmerman and Westerink, 1997). Therefore, activation of SNr GABA-A receptors via $\mathrm{METH}$-induced increases in DA release and D1- 
mediated stimulation of GABA release by METH could disinhibit thalamocortical glutamatergic activity and increase corticostriatal GLU release.

The overarching hypothesis of this study is that METH triggers a polysynaptic process characterized by (1) enhanced striatonigral GABAergic transmission, (2) activation of GABA-A receptors in the SNr leading to decreased nigrothalamic activity, (3) disinhibition of thalamocortical activity, and ultimately, (4) increased corticostriatal GLU release and (5) long-term depletion of striatal DA content. To test this hypothesis, we examined the effects of METH on GAD65 mRNA expression in the striatum and extracellular GABA concentrations in the $\mathrm{SNr}$ and ventromedial thalamus (VM). Moreover, because DA D1 receptors positively modulate GABA release within the $\mathrm{SNr}$, and GABA-A receptors are distributed on nigrothalamic projections, we examined whether an antagonist to D1 or GABA-A receptors into the $\mathrm{SNr}$ would block the hypothesized decreases in extracellular GABA within the VM, the increase in extracellular GLU in the striatum, and the long-term decrease in DA content within the striatum.

\section{Materials and Methods \\ Subjects}

Male Sprague Dawley rats (Harlan Sprague Dawley, Indianapolis, IN) weighing 175-200 gm at the beginning of experimental procedures were housed under a $12 \mathrm{hr}$ light/dark cycle (lights on from 7:00 A.M. to 7:00 P.M. in a temperature-controlled $\left(21-23^{\circ} \mathrm{C}\right)$ and humidity-controlled room. The rats were initially housed four per cage until the day of surgery, on which they were then individually housed. Food and water were available ad libitum. All experimental procedures were performed between 7:00 A.M. and 7:00 P.M. and performed in accordance with the National Institutes of Health Guide for the Care and Use of Laboratory Animals.

\section{Drugs}

$1(S), 9(R)-(-)$-Bicuculline methobromide (BIC) and $R-(+)-7$-chloro8-hydroxy-3-methyl-1-phenyl-2,3,4,5-tetrahydro-1H-3-benzazepine hydrochloride (SCH 23390) were obtained from Sigma-Aldrich (St. Louis, MO). METH was supplied by the National Institute on Drug and Abuse (Research Triangle Park, NC). Doses of METH refer to the weight of the salt. Four injections of METH $(10 \mathrm{mg} / \mathrm{kg})$ were administered intraperitoneally every $2 \mathrm{hr}$. BIC (10 $\mu \mathrm{M})$ and SCH $23390(10 \mu \mathrm{M})$ were administered via reverse dialysis in modified Dulbecco's buffered saline (in mм: $137 \mathrm{NaCl}, 2.7 \mathrm{KCl}, 0.5 \mathrm{MgCl}_{2}, 8.1 \mathrm{Na}_{2} \mathrm{HPO}_{4}, 1.5 \mathrm{KH}_{2} \mathrm{PO}_{4}, 1.2$ $\mathrm{CaCl}_{2}$, and $5 \mathrm{D}$-glucose, $\mathrm{pH} 7.4$ ). SCH 23390 (4 mg) was initially dissolved in $50 \mu \mathrm{l}$ of glacial acetic acid followed by $950 \mathrm{ml}$ of Dulbecco's buffered saline to produce a stock solution. This stock solution was diluted to $10 \mu \mathrm{M}$ with Dulbecco's buffer, and $\mathrm{pH}$ was adjusted to 7.4 with $0.1 \mathrm{~N} \mathrm{NaOH}$. The vehicle control for this perfusion medium was prepared identically but without the SCH 23390.

\section{Experimental procedures}

For the microdialysis experiments, all rats were anesthetized with a combination of xylazine $(12 \mathrm{mg} / \mathrm{kg})$ and ketamine $(80 \mathrm{mg} / \mathrm{kg})$ and placed into a Kopf stereotaxic frame. The skull was exposed, and holes were drilled through the skull above the lateral striatum [anteroposterior (AP), +1.2; mediolateral (ML), \pm 3.2 ; dorsoventral (DV), $-8.2 \mathrm{~mm}$ ], ventromedial thalamus ( $\mathrm{AP},-2.3$; ML, $\pm 1.4 ; \mathrm{DV},-8.4 \mathrm{~mm}$ ), and substantia nigra pars reticulata at a $15^{\circ}$ angle (AP, $-5.6 ; \mathrm{ML}, \pm 4.1 ; \mathrm{DV},-10.2 \mathrm{~mm}$ ) (Paxinos and Watson, 1982). When two probes were implanted, they were on the same side of the brain. One probe was placed in the $\mathrm{SNr}$, and a second probe was placed in either the ipsilateral VM or the ipsilateral striatum. All dialysis probes were of a concentric flow design and constructed as previously described by Yamamoto and Pehek (1990). The lengths of the active dialysis membrane [Spectrapor, 6000 molecular weight cutoff, $210 \mu \mathrm{M}$ optical density (OD)] were as follows: striatum 4 $\mathrm{mm}$; thalamus $1 \mathrm{~mm}$; substantia nigra $1.5 \mathrm{~mm}$. The probes were then lowered to the appropriate position and secured to the skull with three stainless steel screws and cranioplastic cement.

\section{Microdialysis procedures}

The day after surgery, modified Dulbecco's phosphate buffered saline medium was pumped via a dual channel swivel (Instech Laboratories, Plymouth Meeting, PA) through the microdialysis probes with a Harvard Apparatus (Hollison, MA) model 22 syringe infusion pump at a rate of 2 $\mu \mathrm{l} / \mathrm{min}$, as previously described in Matuszewich and Yamamoto (1999). There was a $1.5 \mathrm{hr}$ equilibration period before baseline sample collections. Baseline dialysate samples were collected for $2 \mathrm{hr}$ after which the perfusion medium of the probe inserted in the $\mathrm{SNr}$ was switched to a medium containing either BIC, SCH 23390, or the vehicle perfusion Dulbecco's medium, $\mathrm{pH}$ 7.4. METH or saline was injected after the last baseline sample was collected. Subsequent dialysis samples were collected every hr for $8 \mathrm{hr}$ in the initial experiments to measure GABA within the $\mathrm{SNr}$ during the perfusion of SCH 23390. To enhance the temporal resolution for the detection of possible changes in GABA or glutamate, all subsequent microdialysis experiments in the $\mathrm{SNr}$, ipsilateral ventromedial thalamus, and ipsilateral striatum used sample collections every 30 $\mathrm{min}$. The dead volumes of all probes were calculated so as to synchronize the timing and initiation of drug perfusion for the dual probe microdialysis experiments.

Rectal temperatures were measured $1 \mathrm{hr}$ after each of four injections of either saline or METH.

\section{Biochemical measurements}

$G A B A$. The concentrations of GABA in dialysate samples were determined by HPLC with electrochemical detection, as previously described by Smith and Sharp (1994). GABA was derivatized with O-phthaldialdehyde (OPA) and sodium sulfite. Briefly, $2 \mu \mathrm{l}$ of the stock derivatization reagent containing $22 \mathrm{mg}$ OPA, $9 \mathrm{ml}$ of $0.4 \mathrm{~m}$ boric acid, $\mathrm{pH} 10.4,0.5 \mathrm{ml}$ of $100 \%$ ethanol, and $0.5 \mathrm{ml}$ of $1 \mathrm{M}$ sodium sulfite was added to $20 \mu \mathrm{l}$ of dialysate or standard, vortexed, and allowed to react for $5 \mathrm{~min}$ before injecting onto a C18 column $(100 \times 2.0 \mathrm{~mm}, 3 \mu \mathrm{m}$ particle size; Phenomenex, Torrance, CA). Separation of GABA was achieved with a mobile phase consisting of $0.1 \mathrm{~m}$ sodium phosphate and $0.1 \mathrm{~mm}$ EDTA in $10 \%$ methanol at $\mathrm{pH} 4.4$. GABA was detected with an LC-4B amperometric detector (Bioanalytical Systems, Inc., Lafayette, IN) using a $6 \mathrm{~mm}$ glassy working electrode maintained at a potential of $0.7 \mathrm{~V}$ relative to an $\mathrm{Ag}-\mathrm{AgCl}$ reference electrode.

Glutamate. The concentrations of GLU in dialysate samples were determined by HPLC coupled to fluorescence detection. GLU was derivatized with OPA (Donzanti and Yamamoto, 1988). Briefly, the stock derivatization reagent was prepared by dissolving $27 \mathrm{mg}$ OPA in $9 \mathrm{ml}$ of $0.1 \mathrm{M}$ sodium tetraborate, $\mathrm{pH} 9.4$, and $1 \mathrm{ml}$ of $100 \%$ methanol to which $15 \mu \mathrm{l}$ $\beta$-mercaptoethanol was added. This stock solution was then diluted 1:3 with sodium tetraborate buffer. A $10 \mu$ laliquot of this reagent solution was added to $20 \mu \mathrm{l}$ of dialysate or standard, vortexed, and allowed to react for 1.5 min before injecting onto a C18 column $(100 \times 2.0 \mathrm{~mm}, 3 \mu \mathrm{m}$ particle size; Phenomenex). GLU was eluted using a mobile phase consisting of $0.1 \mathrm{~m}$ sodium phosphate and $0.1 \mathrm{~mm}$ EDTA in $10 \%$ methanol, $\mathrm{pH}$ 6.7. GLU was detected using a Waters 474 Scanning Fluorescence Detector (Milford, MA) with an excitation wavelength $(\operatorname{Ex} \lambda)=340 \mathrm{~nm}$ and emission wavelength $(\operatorname{Em} \lambda)=440 \mathrm{~nm}$.

\section{In situ hybridization}

Separate groups of rats were used for these studies. METH or saline was injected as described above. Five hours after the last injection, rats were killed by rapid decapitation, and whole brains were immediately frozen on dry ice. Sections from the striatum were processed for in situ hybridization histochemistry with riboprobes as previously described by (Nielsen and Soghomonian, 2004). Briefly, 10- $\mu$ m-thick cryostat-cut frozen sagittal sections at the striatal level were produced from saline and METH-treated rats. Sections were quickly dried and fixed in 3.5\% paraformaldehyde followed by prehybridization washes in triethanolamine and tris-glycine. Sections were then hybridized for $4 \mathrm{hr}$ at $52^{\circ} \mathrm{C}$ with 4.0 ng in $20 \mu \mathrm{l}$ of radiolabeled cRNA probe per section (average specific activity: $4.3 \times 10^{5} \mathrm{cpm} / \mathrm{ng}$ ). The $\left[{ }^{35} \mathrm{~S}\right]$-labeled cRNA probe was synthesized from a rat GAD 65 cDNA inserted into bluescript SK plasmid, which was linearized with HindIII. The cRNA probe was diluted in hybridiza- 


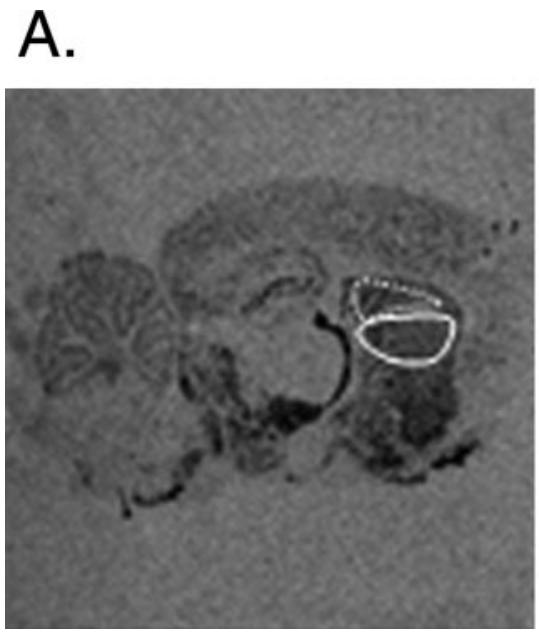

\section{B.}

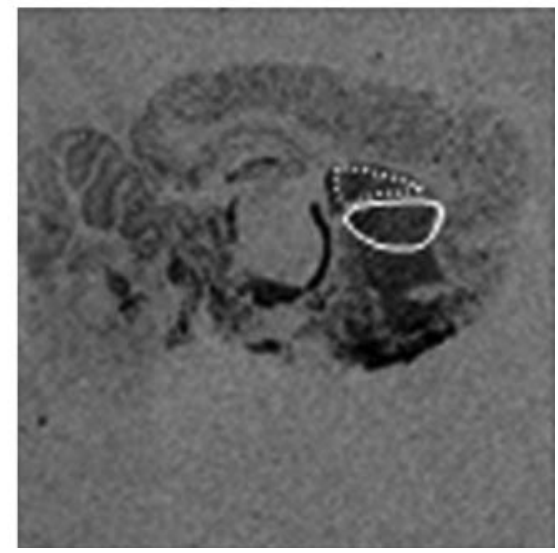

Figure 1. $\quad A, B$, Negative images of $\mathrm{x}$-ray films from sagittal brain sections processed for in situ hybridization with a ${ }^{35} S$-labeled CRNA probe for GAD65 mRNA. A, Saline-control and (B) METH-treated rats. The division of the dorsal and ventral neostriatum is noted by the dotted areas. The quantitative analysis of labeling was performed in the ventral and dorsal neostriatum (denoted by the white dotted areas).
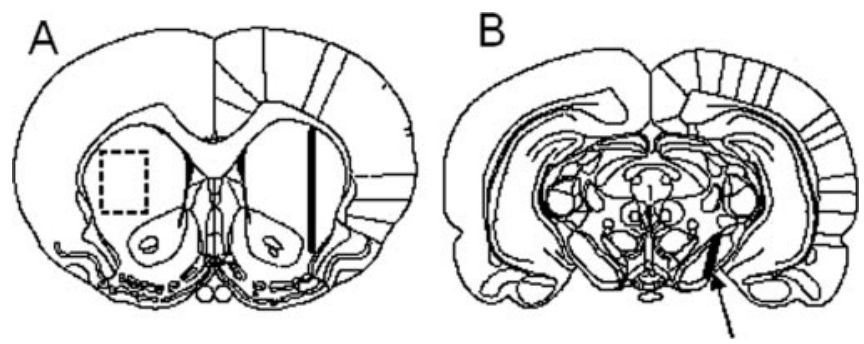

Figure 2. $A, B$, Schematic diagram of probe placement in the lateral striatum $(A)$ and the substantia nigra pars reticulata $(B)$. The regions were (in millimeters relative to bregma): lateral striatum (AP + 1.2) and substantia nigra pars reticulata (AP - 5.6). The black lines represent the probe membrane in the region. The arrow points to the location of the probe in the substantia nigra. The hatched box on the left side of the diagram in $A$ represents the area within the striatum dissected for DA tissue content analysis.

tion solution containing $40 \%$ formamide, $10 \%$ dextran sulfate, $1 \times$ SSC, $10 \mathrm{~mm}$ dithiothreitol, $1.0 \%$ sheared salmon sperm DNA, 1.0\% yeast tRNA, and $1 \times$ Denhardt. The sections were then subsequently washed in $50 \%$ formamide at $52^{\circ} \mathrm{C}$ for 5 and $20 \mathrm{~min}$, RNase $\mathrm{A}(100 \mu \mathrm{g} / \mathrm{ml}$; Sigma-Aldrich) for $30 \mathrm{~min}$ at $37^{\circ} \mathrm{C}$, and in $50 \%$ formamide for $5 \mathrm{~min}$ at $52^{\circ} \mathrm{C}$. Sections were then dehydrated in ethanol and defatted in xylene and apposed to Kodak Biomax-MR x-ray films, exposed in light-tight cassettes for 11-14 d. Films were developed in Kodak D-19 for 3.5 min at $14^{\circ} \mathrm{C}$.

\section{Quantification of labeling}

Levels of GAD65 mRNA labeling in the dorsal and ventral neostriatum were quantified on $\mathrm{x}$-ray films by computerized densitometry with a Macintosh computer, a Sony CCD video camera, and the NIH Image software. The OD of labeling in the striatum was calculated after subtracting the optical density of the film and standardization against emulsion-coated filters (Eastman Kodak, Rochester, NY). The value for each rat was calculated as the average value from three sections. The average level of labeling was then calculated for rats injected with saline or METH.

\section{Tissue content analysis}

Seven days after dialysis, all rats were killed by rapid decapitation, and brains were removed and quick-frozen in dry ice. Brains were sectioned on a cryostat $\left(-20^{\circ} \mathrm{C}\right)$. The striatum on the side ipsilateral and contralateral to the probe placements in the $\mathrm{SNr}$ and VM was dissected from 400 $\mu \mathrm{m}$ coronal sections. Tissue samples were sonicated in $300 \mu \mathrm{l}$ of cold $0.1 \mathrm{~N} \mathrm{HClO}_{4}$ and centrifuged at $14,000 \times g$ for $6 \mathrm{~min}$ at $4^{\circ} \mathrm{C}$. DA was separated on a C18 column $(100 \times 2.0 \mathrm{~mm}, 3$ $\mu \mathrm{m}$ particle size; Phenomenex) and eluted with a mobile consisting of $32 \mathrm{~mm}$ citric acid, 54.3 mM sodium acetate, $0.074 \mathrm{~mm}$ EDTA $\left(\mathrm{Na}_{2}-\right.$ EDTA), $0.215 \mathrm{~mm}$ octyl sodium sulfate, and 3\% methanol, $\mathrm{pH}$ 3.8. Separation of DA and DOPAC was confirmed before each dialysis experiment. Compounds were detected with an LC-4C amperometric detector (Bioanalytical Systems, West Lafayette, IN) with a glassy carbon working electrode maintained at a potential of $+0.670 \mathrm{~V}$ relative to an $\mathrm{Ag}-\mathrm{AgCI}$ reference electrode. Data were recorded using the EZ Chrom (Scientific Software, Pleasanton, CA) software package. Concentrations were expressed as picogram per microgram of protein. Protein content was determined by method of Bradford.

\section{Histology}

All dialysis probe placements in the striatum, $\mathrm{SNr}$, and VM were verified from $40 \mu \mathrm{m}$ corona sections. Only data from experiments with verified probe placements were included in the statistical analysis.

\section{Statistical analysis}

Striatal DA tissue content was analyzed using two-way ANOVA followed by Tukey's post hoc test to determine significant differences between treatment groups. For the dialysis experiments, all data are presented as percentage of baseline to standardize across all the treatment groups and allow for an effective comparison between conditions for GLU and GABA. Changes in amino acid concentrations over time as a function of treatment were analyzed by a two-way ANOVA with repeated measures, using treatment as a between subjects variable and time as a repeated measures variable. For the in situ hybridization studies, optical density measures between two groups were statistically analyzed by a Student's $t$ test. In all cases, a level of $p<0.05$ was considered statistically significant.

\section{Results}

In situ hybridization was used to assess METH-induced changes in striatal GAD65 mRNA levels. Figure 1 illustrates GAD65 mRNA levels as visualized on $\mathrm{x}$-ray films in a saline-treated (Fig. $1 A$ ) and a METH-treated rat (Fig. $1 B$ ). High-dose METH significantly increased GAD65 mRNA levels by 35.6 and $29.7 \%$ in the ventral and dorsal neostriatum, respectively, of rats killed $5 \mathrm{hr}$ after METH compared with saline-treated rats [ventral neostriatum: METH, $0.1440 \pm 0.007$; saline, $0.106 \pm 0.006$ (mean relative OD $\pm \mathrm{SEM}$ ), METH vs saline; $T=3.92$; $p<0.05$; dorsal neostriatum: METH, $0.135 \pm 0.005$; saline, $0.104 \pm 0.007$ (mean relative OD \pm SEM), METH vs saline; $T=3.35 ; p<0.05$ ]. There was no significant difference in METH-induced increases in GAD65mRNA expression between the dorsal and ventral regions of the neostriatum.

In vivo microdialysis experiments were performed to investigate the effects of METH on the GABAergic striatonigral pathway. Figure 2, $A$ and $B$, illustrates the locations of the microdialysis probes. Figure 3 illustrates the effect of high-dose METH on the basal extracellular concentrations of GABA in the SNr. The basal concentration of GABA in dialysate from the $\mathrm{SNr}$ was $26.2 \pm 3.4 \mathrm{pg} / 20 \mu \mathrm{l}$. METH significantly increased extracellular concentrations of GABA in the SNr by $50 \%$. Perfusion of the D1 antagonist SCH $23390(10 \mu \mathrm{M})$ in the $\mathrm{SNr}$ attenuated the METH-induced increases in extracellular GABA. Intranigral perfusion of SCH 23390 alone did not significantly affect basal extracellular concentrations of GABA in the SNr (Fig. 3). 


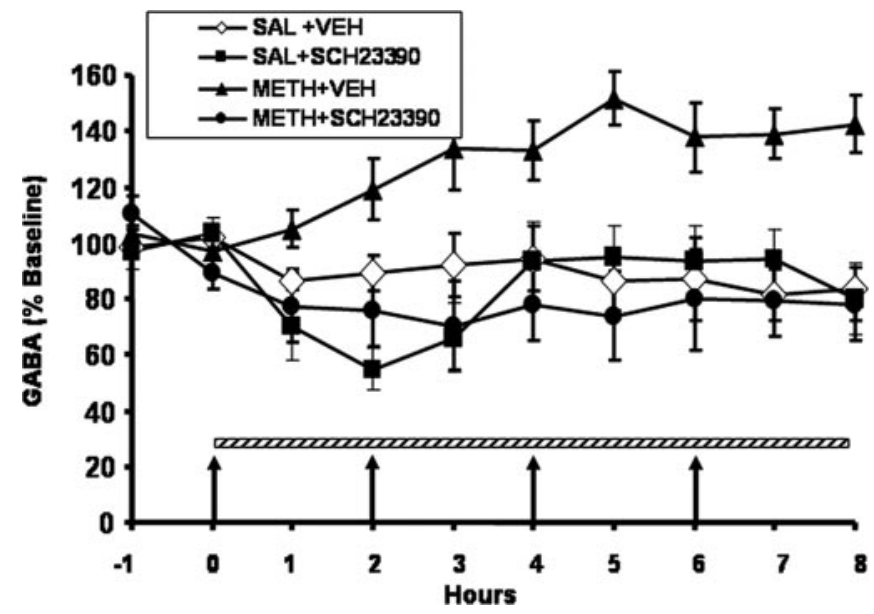

Figure 3. The effects of intranigral perfusion of SCH23390 (10 $\mu \mathrm{M})$ on METH-induced changes in extracellular GABA concentrations in the SNr. Four injections of METH $(10 \mathrm{mg} / \mathrm{kg})$ or $0.9 \%$ saline $(1 \mathrm{ml} / \mathrm{kg}$ ) were given every $2 \mathrm{hr}$ (indicated by arrows). Dialysate samples were collected in the $\mathrm{SNr}$ every hour during the $2 \mathrm{hr}$ baseline period and for the $8 \mathrm{hr}$ drug treatment period. SCH23390 was reverse dialyzed into the SNr after the baseline period and continuously perfused until the end of the experiment (indicated by hatched bar). Data are expressed as mean \pm SEM percentage of baseline values. METH plus vehicle treatment group had a significant increase in extracellular GABA concentrations compared with METH plus SCH23390 (twoway ANOVA with repeated measures, $\mathrm{SCH} 23390$ simple main effect; $\left.F_{(1,72)}=5.17 ; p<0.05\right)$. There is no difference between saline plus vehicle-, saline plus $\mathrm{SCH} 23390-$, and METH plus SCH23390-treated rats. $n=8-9$ rats per group.

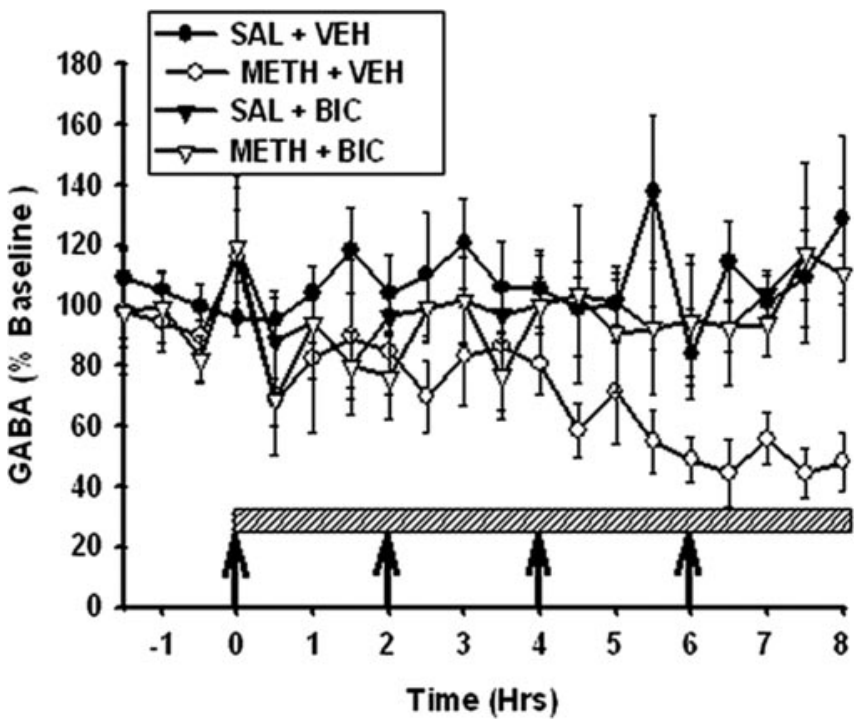

Figure 4. The effects of the intranigral perfusion of the GABA-A receptor antagonist BIC (10 $\mu \mathrm{M})$ on METH-induced changes in extracellular GABA in the VM thalamus. Four injections of METH $(10 \mathrm{mg} / \mathrm{kg}$ ) or $0.9 \%$ saline $(1 \mathrm{ml} / \mathrm{kg}$ ) were given every $2 \mathrm{hr}$ (indicated by arrows). Dialysate samples were collected in the VM every hour during the $2 \mathrm{hr}$ baseline period and the $8 \mathrm{hr}$ drug treatment period. BIC was reverse dialyzed into the $\mathrm{SNr}$ after the baseline period and continuously perfused until the end of the experiment (indicated by hatched bar). Data are expressed as mean \pm SEM percentage of baseline values. METH plus vehicle group showed a significant decrease in extracellular GABA concentrations compared with METH plus BIC (two-way ANOVA with repeated measures, BIC main effect; $\left.F_{(1,379)}=15.34 ; p<0.05\right)$. There was no difference between saline plus vehicle-, saline plus BIC-, and METH plus BIC-treated rats. $n=9-10$ rats per group.

A separate series of experiments was performed to investigate the effect of METH on basal extracellular concentrations of GABA in the VM. The basal concentration of GABA in dialysate from the VM was $25.6 \pm 2.4 \mathrm{pg} / 20 \mu \mathrm{l}$. Figure 4 illustrates the effect of METH on extracellular concentrations of GABA in the

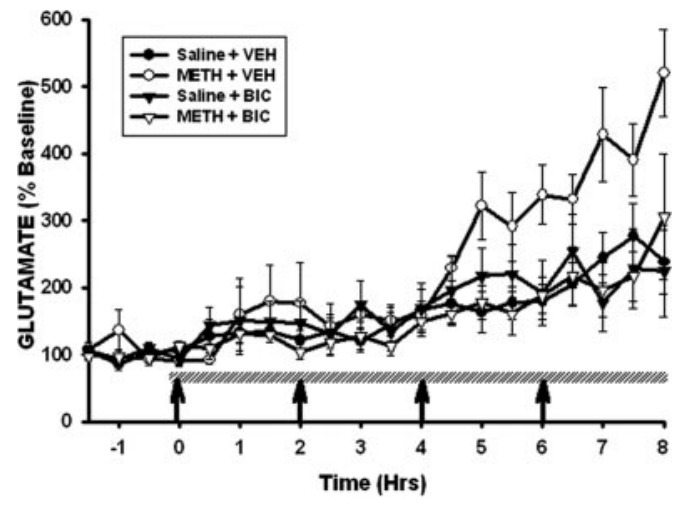

Figure 5. The effects of intranigral perfusion of bicuculline on METH-induced GLU release in the striatum. Four injections of METH $(10 \mathrm{mg} / \mathrm{kg})$ or $0.9 \%$ saline $(1 \mathrm{ml} / \mathrm{kg})$ were given every $2 \mathrm{hr}$ (indicated by arrows). Dialysate samples were collected in the striatum every 30 min during the $2 \mathrm{hr}$ baseline period and the $8 \mathrm{hr}$ drug treatment period. BIC was reverse dialyzed into the $\mathrm{SNr}$ after the baseline period and continuously perfused until the end of the experiment (indicated by hatched bar). Data are expressed as mean \pm SEM percentage of baseline values. METH plus vehicle group showed a significant increase in extracellular GLU concentrations compared with METH plus BIC (two-way ANOVA with repeated measures; BIC main effect; $F_{(1,339)}=7.764 ; p<$ $0.05)$. There was no difference between saline plus vehicle-, saline plus BIC-, and METH plus BIC-treated rats. $n=8-9$ rats per group.

VM. METH significantly decreased extracellular concentrations of GABA in the VM by 35\%. This decrease in extracellular GABA occurred after the third METH injection and was sustained up to $5 \mathrm{hr}$ after the last injection (data not shown). Intranigral perfusion of the GABA-A receptor antagonist bicuculline BIC $(10 \mu \mathrm{M})$ into the $\mathrm{SNr}$ significantly attenuated the METH-induced decrease in extracellular GABA concentrations in the ipsilateral VM (Fig. 4). Intranigral perfusion of BIC alone did not have a significant effect on basal concentrations of extracellular GABA in the ipsilateral VM.

Figure 5 illustrates the effect of intranigral perfusion of BIC on METH-induced increases in extracellular GLU concentrations in the ipsilateral striatum. The basal concentration of GLU in dialysate from the striatum was $1211 \pm 3 \mathrm{pg} / 20 \mu \mathrm{l}$. METH produced a gradual and significantly sustained increase in extracellular GLU concentrations in the lateral striatum. Perfusion of BIC into the $\mathrm{SNr}$ significantly attenuated the METH-induced increases in extracellular concentrations of GLU in the ipsilateral striatum (Fig. 5). Intranigral perfusion of BIC alone did not have a significant effect on basal concentrations of extracellular GLU in the ipsilateral striatum.

Figure 6 illustrates the effect of perfusion of SCH 23390 in the $\mathrm{SNr}$ on striatal tissue concentrations of DA $7 \mathrm{~d}$ after systemic METH administration. METH produced a significant depletion of DA content in striatal tissue. Intranigral perfusion of $\mathrm{SCH}$ 23390 during the administration of METH significantly attenuated the METH-induced depletion of DA content measured $7 \mathrm{~d}$ later in the side ipsilateral to the side of the intranigral perfusion.

Figure 7 illustrates the effect of intranigral perfusion of BIC on striatal tissue concentrations of DA $7 \mathrm{~d}$ after systemic METH administration. METH produced significant depletions in DA tissue content in the striatum contralateral to the side of the intranigral perfusion. In contrast, the perfusion of BIC into the $\mathrm{SNr}$ during the systemic administration of METH completely blocked the long-term METH-induced DA depletions in the striatum ipsilateral to the intranigral perfusion of BIC when measured $7 \mathrm{~d}$ after the microdialysis experiments. Intranigral perfusion of BIC alone did not have a significant effect on DA tissue 


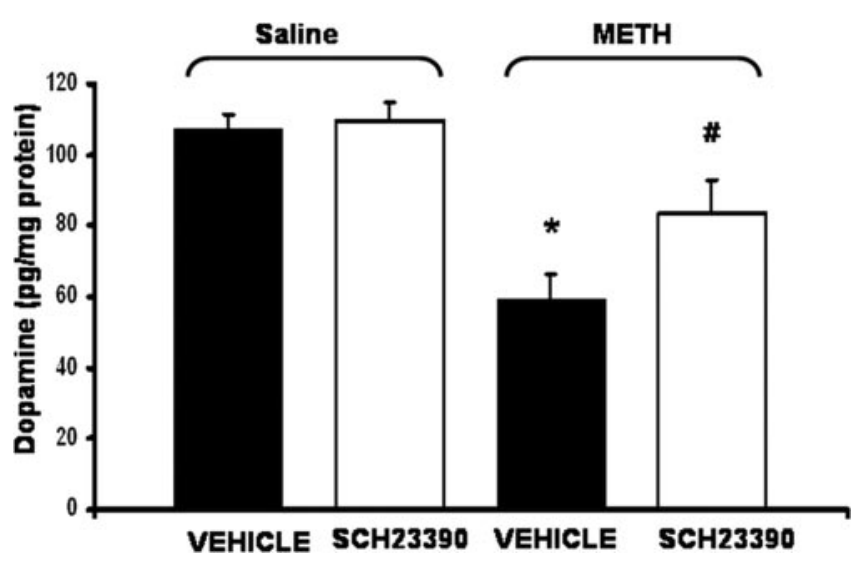

Figure 6. The effect of the intranigral perfusion of the D1 antagonist SCH23390 on long-term METH-induced striatal DA depletions. Rats were killed 1 week after microdialysis. Data are expressed as mean \pm SEM picogram per milligram of protein. Striatal DA content of METH-treated rats was significantly less compared with saline-treated rats $\left(F_{(1,103)}=5.23 ; p<0.05\right)$. Therewas a significant interaction between saline- and METH-administered VEH or SCH23390 $\left(F_{(1,103)}=4.03 ; p<0.05\right)$. Intranigral perfusion of SCH23390 significantly attenuated the METH-induced depletions of DA ( $p<$ 0.05) (METH plus SCH23390 vs METH plus VEH; $T=2.08 ; p<0.05$ ) but did not block the effects of METH because METH plus SCH23390 was still significantly different from saline plus VEH ( $T=2.90$; $p<0.05$ ). There was no difference between saline plus vehicle and saline plus SCH23390-treated rats. ${ }^{*}$ indicates significant $(p<0.05)$ difference from METH plus vehicle and saline plus VEH-treated rats. \# indicates significant $(p<0.05)$ difference from METH plus vehicle and METH plus SCH23390. $n=$ $6-7$ rats per group.

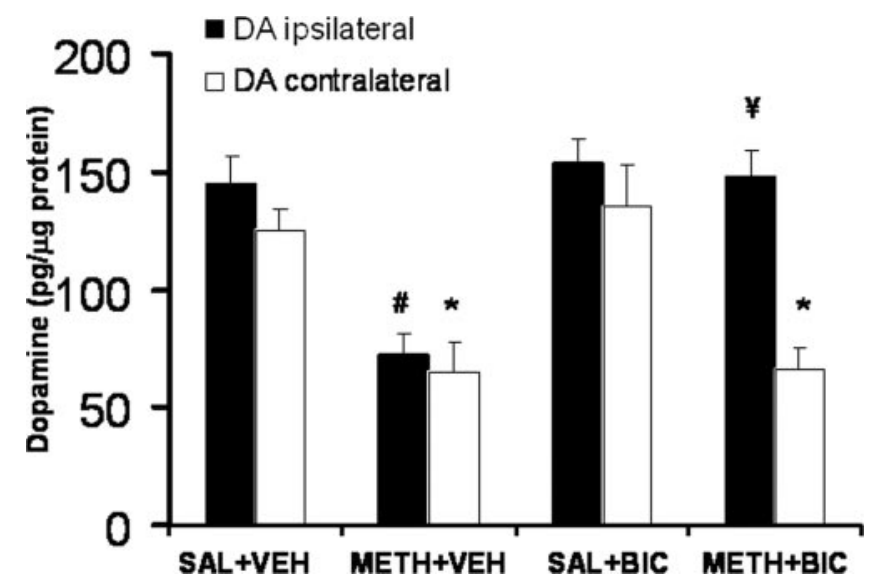

Figure 7. The effects of the intranigral perfusion of BIC on METH-induced striatal DA depletions. Rats were killed 1 week after microdialysis. Data are expressed as mean \pm SEM picogram per milligram of protein. For ipsilateral side: there was a significant main effect of METH $\left(F_{(1,44)}=12.823 ; p<0.05\right), \operatorname{BIC}\left(F_{(1,44)}=15.592 ; p<0.05\right)$, and a significant interaction between METH and BIC $\left(F_{(1,44)}=9.845 ; p<0.05\right)$. Striatal DA content of METH plus vehicletreated rats was significantly less compared with saline-treated rats $(q=6.82 ; p<0.05)$. $¥$ indicates METH plus BIC was higher than METH plus VEH $(q=6.59 ; p<0.05)$. \# indicates significant difference $(p<0.05)$ between METH plus vehicle-, saline plus vehicle-, and saline plus BIC-treated rats. For contralateral side: there was a significant main effect of METH $\left(F_{(1,23)}=75.855 ; p<0.05\right)$. Striatal DA tissue content of METH-treated rats was significantly less compared with saline plus vehicle-treated rats. ${ }^{*}$ indicates significant difference in striatal DA tissue content between SAL plus BIC and METH plus BIC $(q=5.98 ; p<0.05)$ and between SAL plus VEH and METH plus VEH $(q=4.67 ; p<0.05) . n=6-8$ rats per group.

content in the ipsilateral striatum of rats administered systemic saline injections (Fig. 7).

Rectal temperatures were measured at $1 \mathrm{hr}$ after each saline or METH administration. Saline-injected controls had average rectal temperatures of $37.2 \pm 0.2^{\circ} \mathrm{C}$ after each of four injections. The average rectal temperatures of METH-treated rats when measured an hour after each of the four injections was $39.6 \pm 0.4^{\circ} \mathrm{C}$.
Intranigral perfusion of BIC or SCH 23390 in combination with the systemic administration of METH did not affect the hyperthermic rectal temperatures of METH treated rats when measured $1 \mathrm{hr}$ after each of the METH administrations $\left(39.7 \pm 0.2^{\circ} \mathrm{C}\right)$.

\section{Discussion}

Several studies have focused on the roles of DA and GLU in mediating the neurotoxicity of METH to DA nerve terminals, but few studies have examined the possible coordinated interaction between DA and GLU within the basal ganglia circuitry that could explain the long-term depletion of striatal DA content after METH. Although some attention has been directed to the effects of high doses of METH on the striatonigral pathway (Chapman et al., 2001; Hanson et al., 2002; Johnson-Davis et al., 2002), there has been less focus on how activation of this pathway may trigger polysynaptic events culminating in increased extracellular GLU in the striatum. This study elucidates a mechanism of METHinduced GLU release in the striatum that is dependent on both dopaminergic and GABAergic transmission within the striatonigral pathway of the basal ganglia. The findings indicate that METH increases striatonigral GABAergic transmission, as evidenced by GAD65 mRNA expression in the striatum, D1dependent increases in extracellular GABA in the $\mathrm{SNr}$, and subsequent GABA-A receptor-dependent decreases in GABA release in the thalamus and increases in striatal GLU.

The METH-induced increases in striatal GAD65 mRNA expression (Fig. 1) and extracellular GABA within the $\mathrm{SNr}$ (Fig. 3) appear to be mediated by DA within these respective brain regions. GAD65 mRNA expression was used as an index of GABAergic activity within the striatonigral pathway. To our knowledge, this is the first report of specific changes in striatal GAD65 mRNA expression after METH. Although we cannot conclude that increases in striatal GAD65 directly translate into increases in GABA release in the $\mathrm{SNr}$, the increases in striatal GAD65 mRNA may reflect long-term changes in GABAergic activity within the striatonigral pathway after METH. GAD65 gene expression in striatonigral neurons is increased by D1 activation (Laprade and Soghomonian, 1995, 1997). Therefore, METHinduced striatal DA release (Stephans and Yamamoto, 1994) presumably activates striatal D1 receptors to increase GAD65 mRNA expression. This interpretation is consistent with findings that high doses of METH preferentially affect markers of the D1 receptor-associated striatonigral path, as evidenced by changes in striatal preprodynorphin, preproenkaphalin (Wang and McGinty, 1996), and preprotachykinin mRNA (Chapman et al., 2001). Because previous work showed that METH decreases markers of toxicity to DA terminals to a greater extent in the ventral neostriatum (Eisch et al., 1992), a subregional analysis of GAD65 mRNA was conducted. Although METH increased GAD65 mRNA levels in both the ventral and dorsal neostriatum, no differences were observed between the two areas. This may be caused by the fact that more distinct changes are observed in the ventromedial subregion or central sectors more lateral to the areas analyzed in our midline sagittal sections (Eisch et al., 1992).

The increase in GABA after METH is likely mediated by D1 receptors within the $\mathrm{SNr}$. These acute increases in $\mathrm{SNr}$ extracellular GABA were blocked by local perfusion of the D1 antagonist $\mathrm{SCH} 23390$ into the SNr (Fig. 3). Amphetamine increases DA release from dendrites of DAergic neurons in the $\mathrm{SNr}$ (Geffen et al., 1976; Heeringa and Abercrombie, 1995). The increase in extracellular DA can then activate D1 receptors present on striatonigral terminals (Porceddu et al., 1986; Altar and Hauser, 1987) to increase extracellular GABA (Aceves et al., 1995; Timmerman 
and Abercrombie, 1996; Matuszewich and Yamamoto, 1999). It is uncertain if SNr GABA measured in our study originates from striatonigral or pallidonigral terminals. Because D1 antagonism blocks METH-induced increases in extracellular GABA within the $\mathrm{SNr}$ and other findings showing that $\mathrm{SNr}$ D1 receptors are located primarily on striatonigral terminals, METH probably activates the striatonigral GABAergic pathway via striatal GAD65 mRNA and a D1-mediated increase in GABA release from striatonigral terminals.

The major projection from the SNr is to the VM (Somogyi et al., 1979; Bevan et al., 1994). GABA tonically inhibits GABAergic neurons in the SNr via GABA-A receptors (Rick and Lacey, 1994). Additionally, intranigral activation of GABA-A receptors located on GABAergic soma within the $\mathrm{SNr}$ that project to the motor thalamus decrease thalamic neuron firing (Deniau and Chevalier, 1985). Along these lines, the D1 stimulation increased extracellular GABA concentrations in the $\mathrm{SNr}$ and motor activity (Trevitt et al. 2002), the latter presumably mediated through SNr GABAergic neurons that innervate the VM (Faull and Carman, 1968; Beckstead et al., 1979). Therefore, our finding that METH acutely decreases extracellular GABA within the VM (Fig. 5) can be explained by the inhibition of the nigrothalamic pathway resulting from increases in extracellular GABA in the $\mathrm{SNr}$ (Fig. 3). GABAergic neurons of the SNr innervate and inhibit VM neurons (Di Chiara et al., 1979), whereas inhibition of SNr activity by intranigral application of GABA increases the activity of a large percentage of thalamocortical neurons (Deniau et la., 1985). Thus, the METH-induced increase in SNr GABA is probably associated with a decrease in extracellular GABA in the VM mediated by a decrease in impulse flow originating from the activation of SNr GABA-A receptors. This interpretation is supported by the finding that perfusion of the GABA-A antagonist BIC into the SNr blocked the METH-induced decrease in extracellular GABA in the VM (Fig. 4). However, a previous study showed that basal extracellular concentrations of GABA in the VM are insensitive to TTX perfusion in the VM, suggesting that basal extracellular GABA measured by microdialysis in the VM is not impulsederived (Timmerman et al. 1997). The main differences between this study and ours can be explained by the differences between GABA-A receptor-mediated decreases in extracellular GABA originating from the nigrothalamic pathway in the present study and the blockade of sodium channels on all afferents in the VM originating from the globus pallidus, frontal cortex, superior colliculus, and cerebellum (Herkenham, 1979). Regardless, because GABA-A receptor antagonism in the SNr blocked the METHinduced decrease in extracellular GABA in the VM, the decrease in GABA after METH most likely reflects a decrease in GABA-A receptor-mediated and impulse-dependent input to the VM from the $\mathrm{SNr}$.

The decreases in extracellular GABA within the VM after METH (Fig. 5) can alter thalamocortical glutamatergic activity and subsequently, corticostriatal GLU transmission. Because VM glutamatergic neurons innervate the motor cortex (Moran et al., 1982), the METH-induced decrease in GABA in the VM may disinhibit the thalamocortical glutamatergic pathway and increase cortical activity. In fact, METH produces excitotoxicity in the motor cortex, as evidenced by fluorojade immunoreactivity (Eisch et al., 1998) and a long-term decrease in NMDA receptor binding (Eisch et al., 1996). The acute increase in cortical extracellular GLU after METH (Burrows and Yamamoto, 2003) can presumably increase corticostriatal activity and explain the METH-induced increase in extracellular GLU (Nash and Yamamoto, 1992). Moreover, cortical ablation attenuates the
METH-induced increases in extracellular striatal GLU (Burrows and Yamamoto, 2003) and suggests that activation of the corticostriatal glutamatergic pathway plays a role in the excitotoxicity to striatal DA terminals.

METH depletes striatal DA content when measured $7 \mathrm{~d}$ after drug treatment. A disruption of the METH-induced changes in the striatonigral or nigrothalamic pathways was posited to alter the acute METH-induced increases in extracellular GLU in the striatum and consequently, the long-term depletion of striatal DA content. In fact, D1 antagonism attenuated both the acute $\mathrm{METH}$-induced increase in extracellular GABA in the SNr (Fig. 3 ) and the long-term depletions of striatal DA tissue content measured $7 \mathrm{~d}$ later (Fig. 6). In addition to the blockade of the METH-induced decreases in extracellular GABA in the VM (Fig. 4) by BIC perfusion in the $\mathrm{SNr}$, the acute increase in extracellular GLU (Fig. 5) and the subsequent long-term depletion of striatal DA was also blocked on the side ipsilateral to the local perfusion of BIC (Fig. 7). In contrast, intranigral perfusion of SCH 23390 only attenuated but did not completely block the METH-induced DA depletions in striatum (Fig. 6). One explanation is that BIC more directly and effectively alters the nigrothalamic pathway via convergent inputs from the globus pallidus and striatum onto GABA-A receptors, whereas SCH23390 alters D1-mediated GABA release only from striatonigral terminals to affect nigrothalamic GABAergic transmission.

Although D1 antagonism blocked METH neurotoxicity (Sonsalla et al., 1986; O'Dell et al., 1993), the mechanism of this neuroprotection was undefined. Based on the current findings, the neuroprotection by D1 antagonism can be explained at the level of the SNr to acutely attenuate the METH-induced increase in striatonigral GABAergic transmission (Fig. 3), the subsequent maintenance of inhibitory GABAergic tone in the VM (Fig. 4), and the attenuation of the increase in corticostriatal GLU (Fig. 5). These data are consistent with the findings that NMDA receptor antagonists block METH-induced DA neurotoxicity (Sonsalla et al., 1989; Fuller et al., 1992; O’Dell et al., 1992), presumably mediated by ionotropic NMDA and/or AMPA receptors that trigger a cascade of events including calcium-dependent proteolysis (Si-
A.

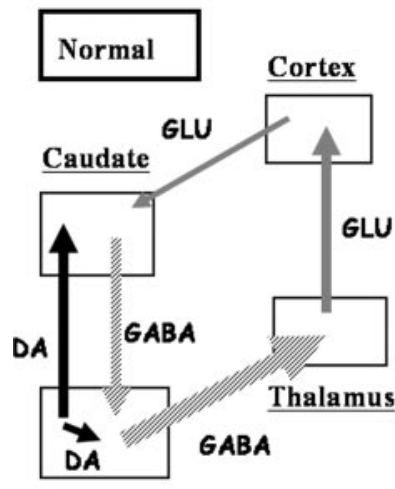

Substantia Nigra
B.

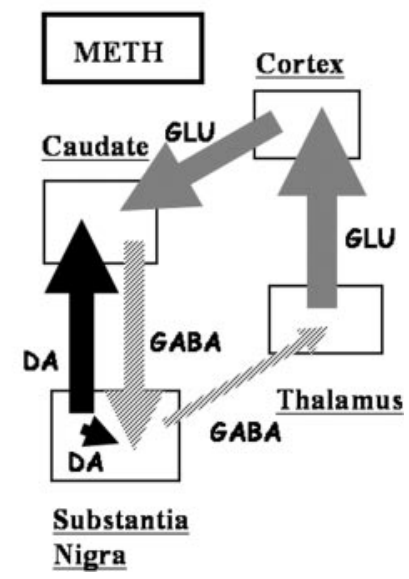

Figure 8. A hypothetical model of polysynaptic effects leading to METH-induced striatal GLU release. Degree of activity is represented by thickness of the arrows. $A$, Normal conditions, under which basal activity of the substantia nigra regulates both DA and GLU release in the striatum. $B$, Effects of METH. DA release in the striatum increases GABAergic release in the SNr, which inhibits nigrothalamic outflow leading to a disinhibition of thalamocortical afferents and subsequent activation of the corticostriatal GLU pathway. 
man and Noszek, 1988) and oxidative stress (Fleckenstein et al., 2000; Burrows and Yamamoto, 2003).

In conclusion, long-term striatal DA depletions produced by METH are, in part, caused by activation of the basal ganglia outflow pathway. Figure 8 illustrates that METH (Fig. $8 \mathrm{~B}$ ) activates the direct striatonigral GABAergic pathway via increased DA release in the striatum and $\mathrm{SNr}$ and activation of $\mathrm{D} 1$ receptors in the $\mathrm{SNr}$ to inhibit nigrothalamic GABA transmission, a subsequent disinhibition of thalamocortical glutamate release, and an eventual increase in corticostriatal GLU.

\section{References}

Aceves J, Floran B, Sierra A, Mariscal S (1995) D-1 receptor mediated modulation of the release of gamma-aminobutyric acid by endogenous dopamine in the basal ganglia of the rat. Prog Neuropsychopharmacol Biol Psychiatry 19:727-739.

Altar CA, Hauser K (1987) Topography of substantia nigra innervation by D1 receptor-containing striatal neurons. Brain Res 410:1-11.

Beckstead RM, Domesick VB, Nauta WJ (1979) Efferent connections of the substantia nigra and ventral tegmental area in the rat. Brain Res 175:191-217.

Bellomo M, Giuffrida R, Palmeri A, Sapienza S (1998) Excitatory amino acids as neurotransmitters of corticostriatal projections: immunocytochemical evidence in the rat. Arch Ital Biol 136:215-223.

Bevan MD, Bolam JP, Crossman AR (1994) Convergent synaptic input from the neostriatum and the subthalamus onto identified nigrothalamic neurons in the rat. Eur J Neurosci 6:320-334.

Burrows KB, Yamamoto BK (2003) Methamphetamine toxicity: roles for glutamate, oxidative processes, and metabolic stress. In: Contemporary clinical neuroscience: glutamate and addiction (Herman BH, ed), pp 211226. Totowa: Humana.

Chapman DE, Hanson GR, Kesner RP, Keefe KA (2001) Long-term changes in basal ganglia function after a neurotoxic regimen of methamphetamine. J Pharmacol Exp Ther 296:520-527.

Deniau JM, Chevalier G (1985) Disinhibition as a basic process in the expression of striatal functions. II. The striato-nigral influence on thalamocortical cells of the ventromedial thalamic nucleus. Brain Res 334:227-233.

Di Chiara G, Porceddu ML, Morelli M, Mulas ML, Gessa GL (1979) Evidence for a GABAergic projection from the substantia nigra to the ventromedial thalamus and to the superior colliculus of the rat. Brain Res 176:273-284.

Donzanti BA, Yamamoto BK (1988) An improved and rapid HPLC/EC method for the isocratic separation of amino acid neurotransmitters from brain tissue and microdialysis perfusates. Life Sci 43:913-922.

Eisch AJ, Gaffney M, Weihmuller FB, O’Dell SJ, Marshall JF (1992) Striatal subregions are differentially vulnerable to the neurotoxic effects of methamphetamine. Brain Res 598:321-326.

Eisch AJ, O’Dell SJ, Marshall JF (1996) Striatal and cortical NMDA receptors are altered by a neurotoxic regimen of methamphetamine. Synapse 22:217-225.

Eisch AJ, Schmued LC, Marshall JF (1998) Characterizing cortical neuron injury with Fluoro-Jade labeling after a neurotoxic regimen of methamphetamine. Synapse 30:329-333.

Faull RL, Carman JB (1968) Ascending projections of the substantia nigra in the rat. J Comp Neurol 132:73-92.

Fleckenstein AE, Gibb JW, Hanson GR (2000) Differential effects of stimulants on monoaminergic transporters: pharmacological consequences and implications for neurotoxicity. Eur J Pharmacol 406:1-13.

Fuller RW, Hemrick-Luecke SK, Ornstein PL (1992) Protection against amphetamine-induced neurotoxicity toward striatal dopamine neurons in rodents by LY274614, an excitatory amino acid antagonist. Neuropharmacology 31:1027-1032.

Geffen LB, Jessell TM, Cuello AC, Iversen LL (1976) Release of dopamine from dendrites in rat substantia nigra. Nature 260:258-260.

Gerfen CR (1989) The neostriatal mosaic: striatal patch-matrix organization is related to cortical lamination. Science 246:385-388.

Hanson GR, Bush L, Keefe KA, Alburges ME (2002) Distinct responses of basal ganglia substance P systems to low and high doses of methamphetamine. J Neurochem 82:1171-1178.

Heeringa MJ, Abercrombie ED (1995) Biochemistry of somatodendritic dopamine release in substantia nigra: an in vivo comparison with striatal dopamine release. J Neurochem 65:192-200.

Herkenham M (1979) The afferent and efferent connections of the ventromedial thalamic nucleus in the rat. J Comp Neurol 183:487-517.

Hotchkiss AJ, Gibb JW (1980) Long-term effects of multiple doses of methamphetamine on tryptophan hydroxylase and tyrosine hydroxylase activity in rat brain. J Pharmacol Exp Ther 214:257-262.

Imam SZ, el Yazal J, Newport GD, Itzhak Y, Cadet JL, Slikker Jr W, Ali SF (2001) Methamphetamine-induced dopaminergic neurotoxicity: role of peroxynitrite and neuroprotective role of antioxidants and peroxynitrite decomposition catalysts. Ann NY Acad Sci 939:366-380.

Johnson-Davis KL, Hanson GR, Keefe KA (2002) Long-term post-synaptic consequences of methamphetamine on preprotachykinin mRNA expression. J Neurochem 82:1472-1479.

Kaneko T, Mizuno N (1988) Immunohistochemical study of glutaminasecontaining neurons in the cerebral cortex and thalamus of the rat. J Comp Neurol 267:590-602.

Kelly E, Jenner P, Marsden CD (1985) The effects of dopamine and dopamine agonists on the release of $3 \mathrm{H}-\mathrm{GABA}$ and $3 \mathrm{H}-5 \mathrm{HT}$ from rat nigral slices. Biochem Pharmacol 34:2655-2662.

Laprade N, Soghomonian JJ (1995) Differential regulation of mRNA levels encoding for the two isoforms of glutamate decarboxylase (GAD65 and GAD67) by dopamine receptors in the rat striatum. Brain Res Mol Brain Res 34:65-74.

Laprade N, Soghomonian JJ (1997) Glutamate decarboxylase (GAD65) gene expression is increased by dopamine receptor agonists in a subpopulation of rat striatal neurons. Brain Res Mol Brain Res 48:333-345.

LaVoie MJ, Hastings TG (1999) Dopamine quinone formation and protein modification associated with the striatal neurotoxicity of methamphetamine: evidence against a role for extracellular dopamine. J Neurosci 19:1484-1491.

Martin LP, Waszczak BL (1994) D1 agonist-induced excitation of substantia nigra pars reticulata neurons: mediation by D1 receptors on striatonigral terminals via a pertussis toxin-sensitive coupling pathway. J Neurosci 14:4494-4506.

Matuszewich L, Yamamoto BK (1999) Modulation of GABA release by dopamine in the substantia nigra. Synapse 32:29-36.

Moran A, Avendano C, Reinoso-Suarez F (1982) Thalamic afferents to the motor cortex in the cat. A horseradish peroxidase study. Neurosci Lett 33:229-233.

Nash JF, Yamamoto BK (1992) Methamphetamine neurotoxicity and striatal glutamate release: comparison to 3,4-methylenedioxymethamphetamine. Brain Res 581:237-243.

Nicholson LF, Faull RL, Waldvogel HJ, Dragunow M (1995) GABA and GABAA receptor changes in the substantia nigra of the rat following quinolinic acid lesions in the striatum closely resemble Huntington's disease. Neuroscience 66:507-521.

Nielsen KM, Soghomonian JJ (2004) Normalization of glutamate decarboxylase gene expression in the entopeduncular nucleus of rats with a unilateral 6-hydroxydopamine lesion correlates with increased GABAergic input following intermittent but not continuous levodopa. Neuroscience 123:31-42.

O'Dell SJ, Weihmuller FB, Marshall JF (1991) Multiple methamphetamine injections induce marked increases in extracellular striatal dopamine which correlate with subsequent neurotoxicity. Brain Res 564:256-260.

O’Dell SJ, Weihmuller FB, Marshall JF (1992) MK-801 prevents methamphetamine-induced striatal dopamine damage and reduces extracellular dopamine overflow. Ann NY Acad Sci 648:317-319.

O'Dell SJ, Weihmuller FB, Marshall JF (1993) Methamphetamine-induced dopamine overflow and injury to striatal dopamine terminals: attenuation by dopamine D1 or D2 antagonists. J Neurochem 60:1792-1799.

Paxinos G, Watson C (1982) The rat brain stereotaxic coordinates. New York: Academic.

Porceddu ML, Giorgi O, Ongini E, Mele S, Biggio G (1986) 3H-SCH 23390 binding sites in the rat substantia nigra: evidence for a presynaptic localization and innervation by dopamine. Life Sci 39:321-328.

Reubi JC, Iversen LL, Jessell TM (1977) Dopamine selectively increases 3HGABA release from slices of rat substantia nigra in vitro. Nature 268:652-654.

Reubi JC, Iversen LL, Jessell TM (1978) Regulation of GABA release by dopamine in the rat substantia nigra. Adv Biochem Psychopharmacol 19:401-404. 
Ricaurte GA, Guillery RW, Seiden LS, Schuster CR, Moore RY (1982) Dopamine nerve terminal degeneration produced by high doses of methylamphetamine in the rat brain. Brain Res 235:93-103.

Rick CE, Lacey MG (1994) Rat substantia nigra pars reticulata neurones are tonically inhibited via GABAA, but not $\mathrm{GABAB}$, receptors in vitro. Brain Res 659:133-137.

Siman R, Noszek JC (1988) Excitatory amino acids activate calpain I and induce structural protein breakdown in vivo. Neuron 1:279-287.

Smith S, Sharp T (1994) Measurement of GABA in rat brain microdialysates using $\mathrm{O}$-phthaldialdehyde-sulphite derivatization and high-performance liquid chromatography with electrochemical detection. J Chromatogr 652:228-233.

Somogyi P, Hodgson AJ, Smith AD (1979) An approach to tracing neuron networks in the cerebral cortex and basal ganglia. Combination of Golgi staining, retrograde transport of horseradish peroxidase and anterograde degeneration of synaptic boutons in the same material. Neuroscience 4:1805-1852.

Sonsalla PK, Gibb JW, Hanson GR (1986) Roles of D1 and D2 dopamine receptor subtypes in mediating the methamphetamine-induced changes in monoamine systems. J Pharmacol Exp Ther 238:932-937.

Sonsalla PK, Nicklas WJ, Heikkila RE (1989) Role for excitatory amino acids in methamphetamine-induced nigrostriatal dopaminergic toxicity. Science 243:398-400.

Stephans SE, Yamamoto BK (1994) Methamphetamine-induced neurotoxicity: roles for glutamate and dopamine efflux. Synapse 17:203-209.
Timmerman W, Abercrombie ED (1996) Amphetamine-induced release of dendritic dopamine in substantia nigra pars reticulata: D1-mediated behavioral and electrophysiological effects. Synapse 23:280-291.

Timmerman W, Westerink BH (1997) Electrical stimulation of the substantia nigra reticulata: detection of neuronal extracellular GABA in the ventromedial thalamus and its regulatory mechanism using microdialysis in awake rats. Synapse 26:62-71.

Trevitt T, Carlson B, Correa M, Keene A, Morales M, Salamone JD (2002) Interactions between dopamine D1 receptors and gamma-aminobutyric acid mechanisms in substantia nigra pars reticulata of the rat: neurochemical and behavioral studies. Psychopharmacology (Berl) 159:229-237.

Wagner GC, Ricaurte GA, Seiden LS, Schuster CR, Miller RJ, Westley J (1980) Long-lasting depletions of striatal dopamine and loss of dopamine uptake sites following repeated administration of methamphetamine. Brain Res 181:151-160.

Wang JQ, McGinty JF (1996) D1 and D2 receptor regulation of preproenkephalin and preprodynorphin mRNA in rat striatum following acute injection of amphetamine or methamphetamine. Synapse 22:114-122.

Yamamoto BK, Pehek EA (1990) A neurochemical heterogeneity of the rat striatum as measured by in vivo electrochemistry and microdialysis. Brain Res 506:236-242.

Yamamoto BK, Zhu W (1998) The effects of methamphetamine on the production of free radicals and oxidative stress. J Pharmacol Exp Ther 287: $107-114$. 